\title{
CONTENIDO DE PIGMENTOS \\ Y ELEMENTOS MINERALES \\ EN WITHANIA FRUTESCENS \\ PAUQUY, SU RELACIÓN \\ CON FACTORES ESTACIONALES \\ Y DE POSICIÓN
}

Por María José Martínez, Eduardo Ferrandis ${ }^{2}$, Joaquín Martín ${ }^{1}$, Antonio Escarré ${ }^{1}$.

\section{SUMMARY}

Seasonal variation, influence of the position and orientation of the branches (factors) were studied in the chemical composition of Withania frutescens organs.

The variables are: chlorophyll content, absorbances quotient, leaves persistance index, and the contents in water and mineral elements.

The statistical technique used for the evaluation of the relative variations of factors, was the canonic analysis.

The season is the most influential factor. With respect to this factor, the leaves show a bigger biochemical activity, and so, a bigger temporaly variation. The stems are less dispersive and show a smaller temporal variation.

1 Departamento de Biología. Fac. Ciencias. Univ. Alicante.

2 Departamento de Bioestadística. Fac. Medicina. Univ. Alicante. 
Next significative factor is the height, getting singular importance in the stem samples, where they differ in three sample heights. In general, the leaves tend to accumulate more contents in the lower parts, while stems distribute them more uniformelly.

The orientation is the less significative factor in the canonic analysis, but all the samples analyzed showed marked differences for the sunny and shady portions.

\section{RESUMEN.}

1.- Se ha estudiado la variación estacional así como la influencia de la posición y orientación de las ramas en la composición química de los órganos de Withania frutescens.

2.-Han sido manejadas como variables, las composiciones químicas en clorofilas, carotenoides (IM), índice de persistencia foliar, contenido hídrico y elementos minerales $\mathrm{N}, \mathrm{P}, \mathrm{Ca}, \mathrm{Mg}$. $\mathrm{Na}$ y $\mathrm{K}$.

3.- Para la ponderación de la variación relativa de los factores época, altura en la planta y exposición de las ramas se ha utilizado la técnica estadística del análisis canónico.

4.-La época es el factor más influyente. Las hojas van a presentar respecto a él una mayor actividad bioquímica, y en consecuencia una variación temporal mayor. Por el contrario los tallos son menos dispersivos, lo que hace que exhiban una menor actividad bioquímica y una menor variación temporal. Es posible ordenar los niveles en la forma Abril, Junio, Julio, Agosto. Noviembre y Febrero son intercalables en esta ordenación; ordenación que pone de manifiesto la influencia de clorofilas, índice de persistencia foliar y elementos potasio y fósforo frente a los contenidos de carotenos magnesio y calcio.

5.-La influencia de la altura sigue en significación a la época y es posible diferenciar perfectamente ramas de la parte alta de la planta y ramas de la parte baja. La altura adquiere singular importancia en las muestras de tallos donde son perfectamente diferenciables las tres zonas inicialmente destacadas. Las hojas, en general, tienden a acumular más contenidos en la parte baja, y tallos los distribuye más uniformemente. 
Para las hojas, en las zonas bajas de la planta, predominan las clorofilas, contenido hídrico y los elementos nitrógeno, fósforo, potasio, calcio y magnesio, y en las altas los carotenos, el índice de persistencia foliar y sodio.

Para los tallos, en las zonas bajas se acumula el calcio, en las medias fósforo, nitrógeno, magnesio y en las altas contenido hídrico, potasio y sodio.

6.-En orientación, aún tratándose del factor menos significativo en el análisis canónico, todas las porciones analizadas presentaron diferencias claras para las de sombra y para las de sol. En las muestras de hojas tomadas en zonas de sombra predominan clorofilas, contenido hídrico y elementos nitrógeno, calcio, magnesio, potasio, fósforo y en las soleadas carotenoides, índice de persistencia foliar y sodio. En las muestras de tallos las zonas de sombra acumulan calcio y fósforo y las soleadas nitrógeno, potasio, magnesio, sodio y contenido hídrico.

\section{INTRODUCCIÓN}

En un trabajo anterior (Martín y Escarre, 1980), dábamos a conocer la fenología y algunos aspectos sobre las variaciones en contenidos hídricos, pigmentos y nutrientes minerales en hojas de cuatro arbustos (Withania frutescens, Lycium intricatum, Asparagus albus y Rhamnus lycioides spp. lycioides) que forman parte de un espinar xérico, que se desarrolla en el Cabo de Santa Pola (Alicante), perteneciente, muy probablemente, a la Asociación Chamaeropo-Rhamnetum lycioides O. de Bolós, 1957.

Por otro lado, la existencia de diferencias en cuanto a contenidos hídricos, nutrientes, etc., en árboles y arbustos, debido a la altura de las ramas y orientación (Leroy, 1968; Kramer y Kozlowski, 1979), nos llevó a la elección de una de las especies anteriormente citadas, Withania frutescens, para la realización del presente trabajo.

La finalidad de este estudio es la evaluación de las posibles interferencias entre los factores altura de ramas y orientación, respecto a las variaciones estacionales, en cuanto a los contenidos de agua y nutrientes de tallos y hojas, así como de pigmentos en éstas. 
La evaluación de estos contenidos químicos sirve para constatar el carácter caducifolio estival de la especie. Los resultados obtenidos entran en la misma línea de variación de los trabajos consultados en la bibliografía.

\section{MATERIAL Y MÉTODOS}

Las variables consideradas han sido el contenido en clorofilas, el cociente de absorbancias a 430 y $664 \mathrm{~nm}$., IM (Marfalef, 1974), índice de persistencia foliar, contenido hídrico y el de los elementos minerales $\mathrm{N}, \mathrm{P}, \mathrm{Ca}, \mathrm{Mg}, \mathrm{Na}, \mathrm{y} \mathrm{K}$, expresados en $\%$ respecto a peso seco de material.

Se han seleccionado seis muestreos que corresponden a las fechas: 27 de Noviembre de 1979, 14 de Febrero de 1980, 24 de Abril de 1980, 4 de Junio de 1980, 1 de Julio de 1980 y 5 de Agosto de 1980. En cada una de las épocas se tomaron seis submuestras según los otros dos factores, es decir, según perteneciesen a las zonas alta, media y baja, para sombra y para sol.

Por tanto en el problema que nos ocupa se consideran tres factores, la época del muestreo, la altura a que se tomen las muestras y la orientación con relación a su exposición al sol. Los niveles para esos factores, es decir las distintas épocas, alturas y exposiciones, ya se han especificado anteriormente y sobre cada muestra se han medido el conjunto de variables ya mencionadas.

La elaboración estadística de nuestros datos se ha desarrollado a través del análisis canónico.

Con esta técnica (Rao, 1952; Seal, 1964; Cuadras, 1974) se pretende:

$1^{\circ}$.- Valorar la influencia de los distintos factores sobre el conjunto de variables considerado. Esto se realiza,

a) contrastando la hipótesis estadística de que los distintos niveles de un determinado factor, presentan distribuciones de datos equivalentes,

b) estableciendo criterios de distancia, proximidad o equivalencia entre los distintos niveles de los factores considerados, lo que lleva en algunos casos a la clasificación en grupos o a una ordenación posible de dichos niveles. 
$2^{\circ}$.- Valorar las posibles interferencias entre los factores considerados, es decir si la influencia de un determinado factor sobre el conjunto de variables depende o no de los niveles que presenten otro o los restantes factores.

$3^{\circ}$.-Determinar la importancia relativa con que las variables consideradas influyen en la discriminación de los diferentes niveles.

Los problemas descritos en los apartados $1^{\circ}$ a) y $2^{\circ}$ se formalizan matemáticamente a través del análisis de la varianza múltiple, MANOVA. La estimación matricial generalizada de las varianzas-covarianzas entre las variables consideradas, se descompone mediante este análisis en varias matrices, que condensan y aislan las influencias de los diferentes factores y las posibles interferencias, y una matriz básica que estima las varianzas-covarianzas independientemente de la posible influencia de los factores y sus interferencias. Comparando las matrices correspondientes a los distintos factores e interferencias con la matriz básica se puede determinar, con cierto nivel de seguridad (aquí hemos empleado siempre 0.95), la posible influencia significativa de esos factores y sus interferencias. La comparación se establece mediante el empleo de un estadístico adecuado, en nuestro caso, el estadístico de Wilks.

La formalización matemática de los apartados $1^{\circ} \mathrm{b}$ ) y $3^{\circ}$ constituye el objeto del análisis canónico propiamente dicho. Este análisis obtiene unos nuevos ejes canónicos que, siendo variables aleatorias independientes, maximizan el poder de discriminación sobre los niveles de los factores en estudio o la detección de las posibles interferencias. Estos ejes son precisamente los autovectores de las respectivas matrices con respecto a la matriz básica.

Sobre estos ejes pueden representarse círculos o esferas de confianza para los efectos medios de los distintos niveles en cada factor.

Finalmente, calculando las correlaciones entre los principales ejes significativos y las primitivas variables consideradas puede llegarse a una interpretación lógica de dichos ejes, y a una valoración del aporte de las diferentes variables a la discriminación de los niveles de los factores en estudio. 


\section{RESULTADOS.}

Como primer objetivo nos hemos planteado el estudio de las variables clorifila a y clorofila $b$, ambas magnitudes expresadas en mgr. $\mathrm{l}^{-1}$ (Ziegler y Egle, 1965), conjuntamente con los carotenos, reflejados a través del índice de Margalef (IM).

El número de observaciones de cada variable atiende al hecho de que las muestras de hoja donde han sido analizados los pigmentos se encuentran por duplicado, lo que conduce a un número de observaciones de 12 para el factor época, 24 para altura y 36 para exposición.

\section{EJES CANÓNICOS Y SIGNIFICACIÓN.}

Como resultados del análisis canónico se han obtenido unos autovectores o vectores propios, expresados en forma de matrices, cuya importancia de cara a representación e interpretaciones viene reflejada en los autovalores o valores propios. La visualización numérica de los ejes canónicos permite un estudio de la correspondiente significación, esto es ver si realmente tienen poder discriminatorio en relación al factor considerado. Este estudio requiere la utilización del siguiente estadístico.

$$
F\left(v_{i}, v\right)=\frac{v}{v_{i}} W
$$

siendo, $\mathrm{v}_{\mathrm{i}}$ los grados de libertad del factor en consideración, (época 5, altura 2, exposición 1).

v los grados de libertad de la matriz básica que varía según el análisis.

W autovalor de cada uno de los ejes en cada factor.

Posteriormente una comparación con el estadístico tabulado de Snedecor (Parker, 1976) con nivel de significación del 0.05, pondrá de manifiesto qué ejes canónicos son los que realmente ofrecen una discriminación de factores.

Las tablas 1 y 2 muestran, respectivamente, los valores numéricos de los autovalores y del estadístico F, para cada factor. El doble aste- 
risco, situado en algunos resultados de la tabla 2 , indica aquellos valores de $\mathrm{F}$ que superan la significación del 0.05 y con un solo asterisco están aquellos que, superando dicha significación, no la rebasan holgadamente.

TABLA 1.-Autovalores de los ejes canónicos en el análisis multivariante de pigmentos.

\section{FACTORES}

\section{EJES}

$\mathrm{w}_{1}$
$\mathrm{~W}_{2}$
$\mathrm{~W}_{3}$
ÉPOCA

2.673

0.432

0.072
ALTURA

0.621

0.015

$0.763+10^{-9}$
EXPOSICIÓN

$$
0.275
$$

$-0.138 \cdot 10^{-7}$

$-0.611 \cdot 10^{-9}$

TABLA 2.-Estadístico $\mathrm{F}\left(\mathrm{V}_{\mathrm{i}}, \mathrm{v}\right)$ calculado, en el análisis de pigmentos; $\mathrm{v}=36$.

\section{FACTORES}

$\begin{array}{cccl}\text { EJES } & \text { ÉPOCA } & \text { ALTURA } & \text { EXPOSICIÓN } \\ \mathrm{W}_{1} & 19.25^{* *} & 11.17^{* *} & 9.90^{* *} \\ \mathrm{~W}_{2} & 3.11^{*} & 0.26 & 0 \\ \mathrm{~W}_{3} & 0,52 & 0 & 0\end{array}$

\section{COORDENADAS CANÓNICAS.}

El hecho de que para altura y exposición sólo sea significativo un eje canónico limita las representaciones a una línea recta y, en consecuencia, las coordenadas a un único valor; mientras que para época las representaciones corresponden a un plano y las coordenadas a un par de valores, un punto en el plano. Los valores de las coordenadas, pertenecientes una a cada nivel dentro de los factores, están recogidos en la tabla 3 . 
TABLA 3.-Coordenadas canónicas, pertenecientes a los ejes significativos, en la representación para pigmentos.

\begin{tabular}{|c|c|c|c|c|}
\hline \multirow[b]{2}{*}{ FACTOR } & \multicolumn{4}{|c|}{ COORDENADAS } \\
\hline & NIVEL & EJE $W_{1}$ & EJE $\mathbf{W}_{2}$ & RADIO \\
\hline ÉPOCA & 1 & 0.160 & 0.341 & 0.57 \\
\hline ““ & 2 & -0.242 & -0.229 & “" \\
\hline “" & 3 & 0.410 & -0.524 & “" \\
\hline “" & 4 & 0.002 & 0.073 & “" \\
\hline “ & 5 & -0.659 & -0.007 & “ \\
\hline “ & 6 & -1.618 & -0.164 & “، \\
\hline ALTURA & A & -0.356 & & 0.40 \\
\hline “ & M & 0.345 & & “ \\
\hline “" & B & 0.745 & & “، \\
\hline EXPOSICIÓN & $\mathrm{N}$ & 0.642 & & 0.33 \\
\hline “" & $\mathrm{S}$ & -0.100 & & “" \\
\hline
\end{tabular}

\section{RADIO DE LOS FACTORES.}

Una vez establecidas las representaciones canónicas se marcan unas regiones (círculos de confianza) para cada uno de los niveles, lo cual conduce a establecer posibles agrupaciones según estén o no solapadas dichas regiones. El trazado de estas circunferencias supone el cálculo de su radio a partir de la expresión

$$
r=\frac{1.96}{\sqrt{\mathrm{n}_{\mathrm{i}}}}
$$

donde $n_{i}$ corresponde al número de observaciones de cada variable en el factor considerado. Los radios calculados para los tres factores aparecen en la última columna de la tabla 3 .

Es preciso indicar que la fórmula anterior tiene validez únicamente en aquellos casos en que el número de variables primitivas sea inferior al tamaño de muestras. Para los casos desfavorables supondrá que 
el análisis canónico como tal, no es aplicable al conjunto de variables en estudio, teniendo que reducir éste a un número $\mathrm{p}$, tal que satisfaga

$$
\mathrm{P} \leq \mathrm{n}_{\mathrm{i}}-1
$$

En el estudio concreto de los pigmentos no se precisa la aplicación de (1); la representación de los valores retenidos en la tabla 3 corresponde a la figura 1 .

Para el análisis multivariante del resto de variables en muestras de hoja han sido analizados los porcentajes de los elementos agua, nitrógeno, fósforo, calcio, magnesio, sodio y potasio, así como los índices I.P.F. y V.P.F., como cocientes entre peso seco de hoja y peso seco de tallo y entre peso seco de hoja y peso seco total de hoja y tallo, respectivamente.

El número de observaciones de cada variable es para cada uno de los factores respectivamente 6,12 y 18 . Dado que el número total de variables supera para época la consideración (1), hay que reducir el conjunto de variables. Se ha creído conveniente el recurso de efectuar dos análisis canónicos distintos:

—el primero de ellos analizará las variables agua, magnesio, potasio y V.P.F., y serán interpretados sus resultados para cada época.

—el segundo analizará la totalidad de las variables inicialmente citadas y su interpretación será válida de acuerdo a los otros dos factores, altura y exposición.

La elección de las nuevas variables para época se ha hecho a partir de las correlaciones calculadas entre las distintas variables. Éstas se calculan a partir de la matriz básica, mediante la expresión

$$
r\left(V_{i}, V_{j}\right)=\frac{s_{i j}}{\left|S_{i}\right|\left|s_{j}\right|}
$$



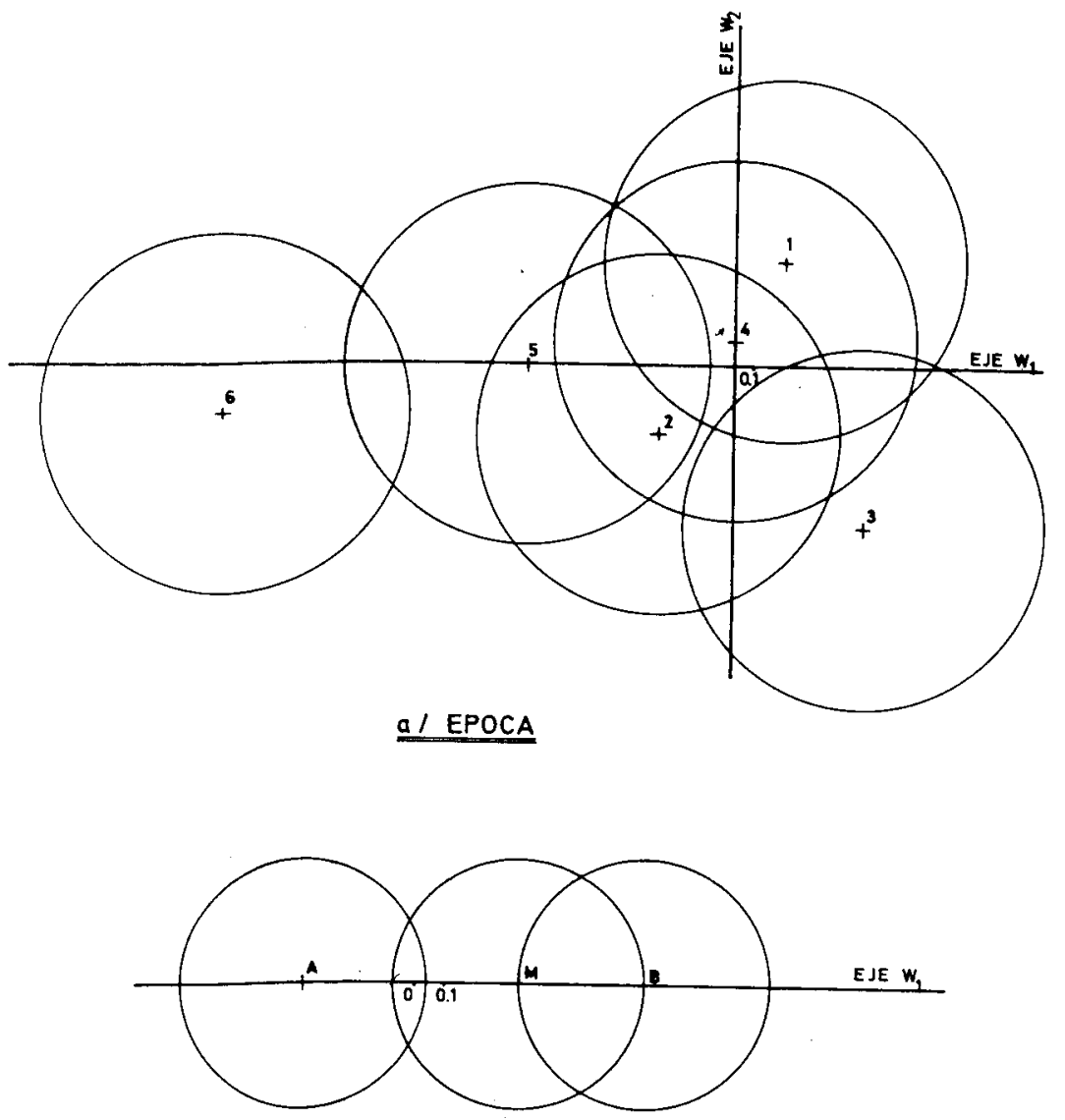

bI ALTURA

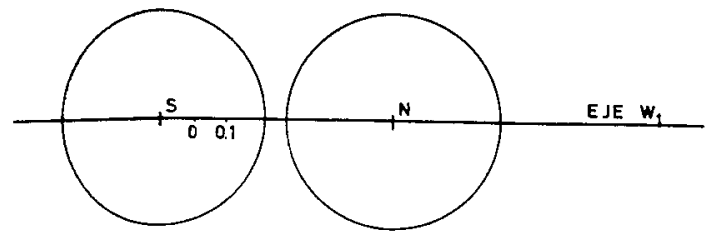

Figura 1.- Representación canónica para pigmentos según los distintos factores 
donde $V_{i}$ y $V_{j}$ representan las variables para las que se calcula la correlación. $S_{i j}$ el elemento de fila i y columna $j$ en la matriz básica. $\left|S_{\mathrm{i}}\right| S_{\mathrm{j}} \mid$ las raíces de los elementos diagonales de dicha matriz que estiman las desviaciones típicas de dichas variables.

Los resultados obtenidos de la aplicación de (2), están recogidos en la tabla 4 para las muestras de hoja y en la tabla 5 para las de tallo. Las cantidades marcadas con un asterisco superan la significación de 0.05 .

Atendiendo al orden establecido en el análisis multivariante de pigmentos, las tablas 6 y 7 contienen los valores numéricos de los ejes canónicos, 8 y 9 los correspondientes estadísticos calculados, y 10 las coordenadas canónicas de los ejes que hayan sido significativos de acuerdo a 8 y 9 y los correspondientes radios. La representación de la tabla 10 está contenida en la figura 2.

TABLA 4.-Correlaciones entre variables en el análisis de hojas.

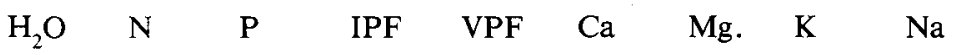

\begin{tabular}{|c|c|c|c|c|c|c|c|c|}
\hline $\mathrm{H}_{2} \mathrm{O}$ & $0.69^{*}$ & 0.29 & 0.09 & -0.02 & 0.29 & 0.10 & 0.46 & $0.69^{*}$ \\
\hline $\mathrm{N}$ & & 0.27 & 0.17 & 0.20 & -0.21 & -0.01 & 0.62 & 0.21 \\
\hline $\mathbf{P}$ & & & 0,05 & -0.05 & 0.33 & $-0.67^{*}$ & 0.39 & 0.29 \\
\hline IPF & & & & $0.92^{*}$ & -0.04 & 0.20 & -0.33 & 0.27 \\
\hline VPF & & & & & -0.10 & 0.22 & -0.39 & 0.12 \\
\hline $\mathrm{Ca}$ & & & & & & $0.67^{*}$ & -0.24 & 0.58 \\
\hline $\mathrm{Mg}$ & & & & & & & -0.15 & 0.39 \\
\hline $\mathrm{K}$ & & & & & & & & -0.19 \\
\hline
\end{tabular}


TABLA 5.-Correlaciones entre variables en el análisis de tallos. $\begin{array}{lllllll}\mathrm{H}_{2} \mathrm{O} & \mathrm{N} & \mathrm{P} & \mathrm{Ca} & \mathrm{Mg} . & \mathrm{K} & \mathrm{Na}\end{array}$

$\begin{array}{lrrrrrr}\mathrm{H}_{2} \mathrm{O} & 0.03 & 0.78^{*} & -0.53 & 0.62 & 0.78^{*} & 0.71^{*} \\ \mathrm{~N} & & 0.11 & 0.29 & 0.39 & 0.12 & -0.04 \\ \mathrm{P} & & & -0.68^{*} & 0.63 & 0.63 & 0.44 \\ \mathrm{Ca} & & & & -0.09 & -0.10 & -0.13 \\ \mathrm{Mg} & & & & & 0.79^{*} & 0.64 \\ \mathrm{~K} & & & & & & 0.57 \\ \mathrm{Na} & & & & & & \end{array}$

TABLA 6.-Autovalores de los ejes canónicos en el análisis multivariante de hojas. Factor época.

\section{EJES}

$$
\begin{aligned}
& W_{1} \\
& W_{2} \\
& W_{3} \\
& W_{4}
\end{aligned}
$$

Factor Época
0.079
0.357
1.839
6.110

TABLA 7.-Autovalores de los ejes canónicos en el análisis multivariante completo de hoja. Restantes factores.

\section{FACTORES}

\section{EJES}

$\mathrm{W}_{1}$

$\mathrm{W}_{2}$

$\mathrm{W}_{3}$

$\mathrm{W}_{4}$

$\mathrm{W}_{5}$

$\mathrm{W}_{6}$

$\mathrm{W}_{7}$

$\mathrm{W}_{8}$

$\mathrm{W}_{9}$
ALTURA

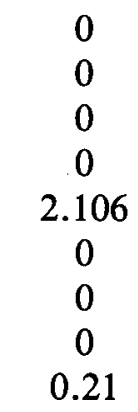

EXPOSICIÓN

0
0
0
0
0
1.61
0
0
0


TABLA 8.-Estadístico $F\left(v_{i}, v\right)$ calculado para el factor época en el análisis de hojas: $\mathrm{v}=10$.

\section{EJES}

$$
\begin{aligned}
& W_{1} \\
& W_{2} \\
& W_{3} \\
& W_{4}
\end{aligned}
$$

FACTOR ÉPOCA

$$
\begin{gathered}
0.16 \\
0.71 \\
3.68^{*} \\
12.22^{* *}
\end{gathered}
$$

TABLA 9.-Significado de los ejes canónicos. Estadístico $F\left(v_{i}, V\right)$, calculado, $\mathrm{v}=10$.

\section{FACTORES}

\section{EJES}

$\mathrm{W}_{1}$
$\mathrm{~W}_{2}$
$\mathrm{~W}_{3}$
$\mathrm{~W}_{4}$
$\mathrm{~W}_{5}$
$\mathrm{~W}_{6}$
$\mathrm{~W}_{7}$
$\mathrm{~W}_{8}$
$\mathrm{~W}_{9}$
ALTURA

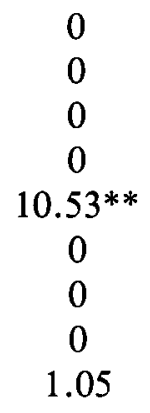

EXPOSICIÓN

0
0
0
0
0
$16.10^{* *}$
0
0
0


TABLA 10.-Coordenadas pertenecientes a los ejes significativos en la representación canónica para hojas.

\section{COORDENADAS}

\begin{tabular}{ccccc} 
FACTOR & NIVEL & EJE W $_{\mathbf{4}}$ & EJE W & RADIO \\
\hline EPOCA & 1 & -2.740 & -5.448 & 0.80 \\
$»$ & 2 & -2.640 & -5.413 & $»$ \\
$»$ & 3 & -0.540 & -4.933 & $»$ \\
$»$ & 4 & -1.133 & -4.552 & $»$ \\
$»$ & 5 & -1.822 & -4.092 & $»$ \\
$»$ & 6 & -3.504 & -4.112 & $»$
\end{tabular}

\section{ALTURA}

$\gg$

$\gg$

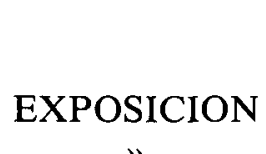

»
A

M

B

N

\section{EJE $\mathbf{W}_{5}$}

$-9.636$

0.57

$-11.080$

$-11.620$

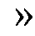

»

EJE $\mathbf{W}_{6}$

4.269

2.475
)

)

El análisis químico de muestras de tallo únicamente se ha realizado sobre los elementos nitrógeno, fósforo, calcio, magnesio, sodio y potasio, así como sobre el contenido hídrico, expresados en $\%$. Son requeridos los dos tipos de análisis estadístico:

-en primer lugar y de acuerdo con el factor época, con número de observaciones igual a 6, serán analizados agua, nitrógeno, calcio y magnesio, estimadas a partir de (2) y reseñadas en la tabla 5,

- a continuación, para el estudio estadístico de los otros dos factores, altura y exposición con número de observaciones igual a 12 y 18 respectivamente, el análisis se efectuará con el conjunto ya establecido de las siete variables. 


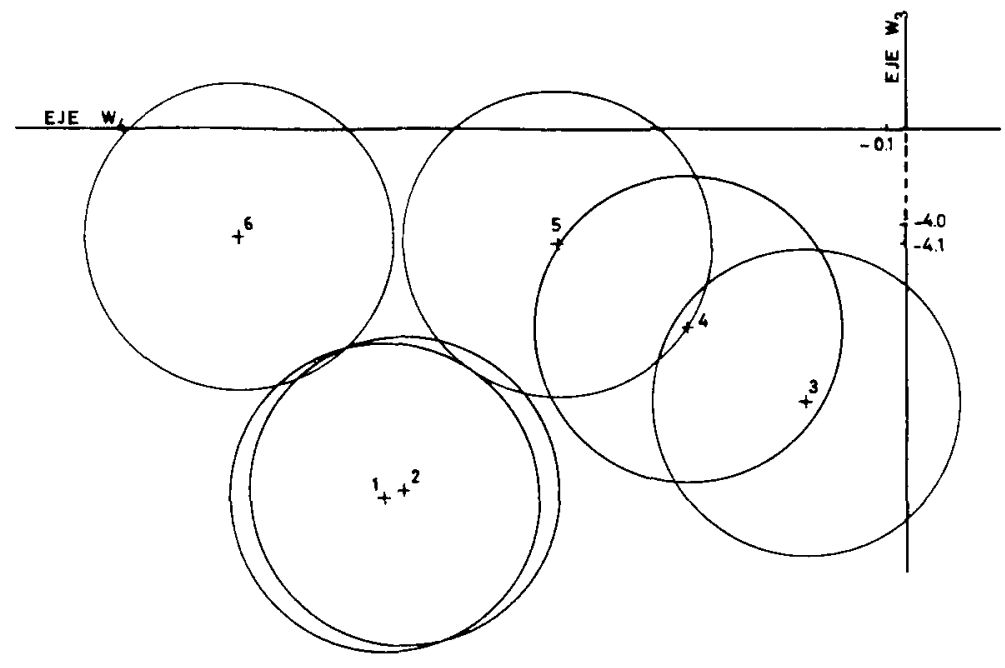

a 1 EPOCA

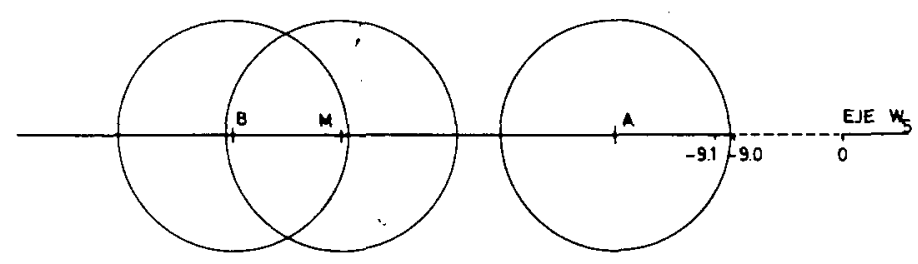

DI ALTURA

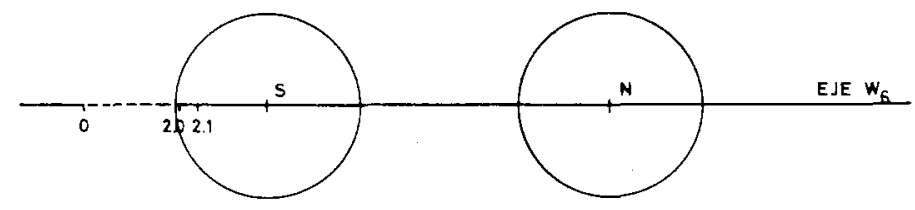

Figura 2.- Representación canónica para el resto de variables en hojas según los distintos factores 
Las tablas $11,12,13,14$ y 15 recogen toda la información obtenida del análisis canónico de tallos para cada uno de los factores. La representación oportuna de tales resultados es la figura 3.

TABLA 11.-Autovalores de cada uno de los ejes canónicos en el análisis multivariante para tallos.

\section{EJES}

$$
\begin{aligned}
& \mathrm{W}_{1} \\
& \mathrm{~W}_{2} \\
& \mathrm{~W}_{3} \\
& \mathrm{~W}_{4}
\end{aligned}
$$

FACTOR ÉPOCA

0.066

0.141

0.944

5.449

TABLA 12.-Autovalores de los ejes canónicos en el análisis multivariante completo de tallos.

\section{FACTORES}

\section{EJES}

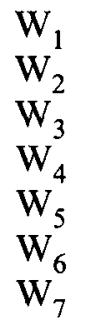

ALTURA

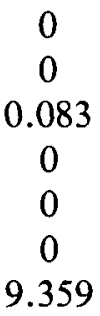

EXPOSICIÓN

0

0

0

0

0

0

2.097

TABLA 13.-Estadístico $F\left(v_{i}, v\right)$ calculado en el análisis de tallos para factor época; $v=10$.

\section{EJES}

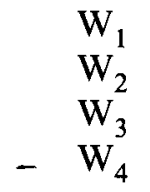

FACTOR ÉPOCA

0.13

0.28

1,89

$10.90^{* *}$ 
TABLA 14.-Estadístico $F\left(v_{i}, v\right)$ calculado, $v=10$. Análisis multivariante de tallos. Restantes factores.

\section{FACTORES}

\section{EJES}

$\mathrm{W}_{1}$
$\mathrm{~W}_{2}$
$\mathrm{~W}_{3}$
$\mathrm{w}_{4}$
$\mathrm{~W}_{5}$
$\mathrm{~W}_{6}$
$\mathrm{w}_{7}$
ALTURA

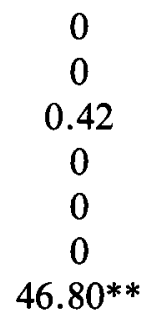

EXPOSICIÓN

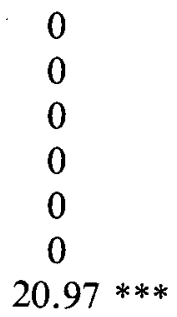

TABLA 15.-Coordenadas pertenecientes a los ejes significativos en la representación canónica para tallos.

\section{COORDENADAS}

FACTOR

ÉPOCA

"

“

“

"

“
NIVEL

1

2

3

4

5

6

ALTURA

"،

“6

EXPOSICIÓN

A

M

B

N

،

EJE $\mathbf{W}_{4}$

RADIO

$-6.574$

$-6.665$

$-7.726$

$-7.159$

$-5.571$

$-4.873$

EJE $\mathbf{W}_{7}$

$-27.11$

$-29.64$

$-31.42$

$-27.60$

$-25.55$

$\mathrm{S}$

0.57

0.80
6
6
6
6
6

،

6

0.46 


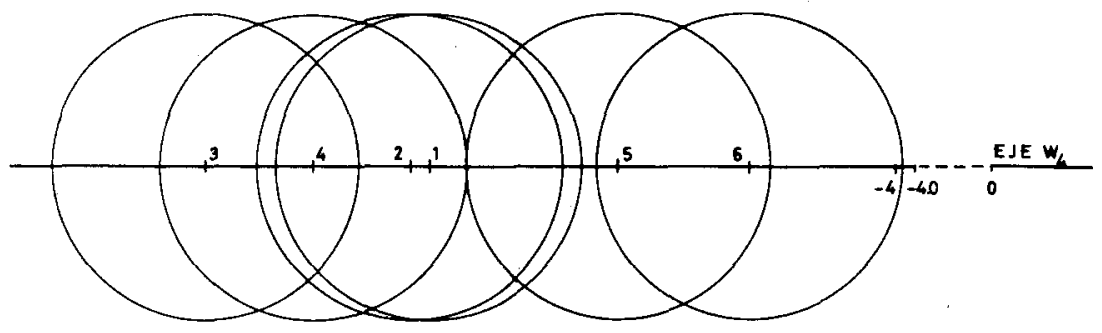

a) EPOCA

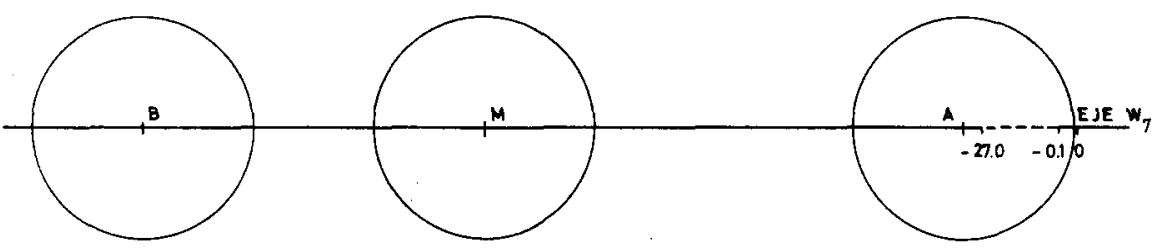

DI ALTURA

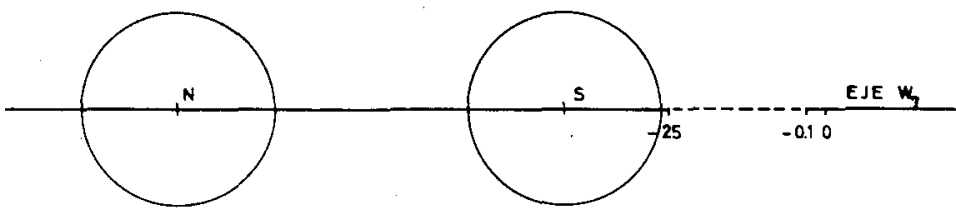

Figura 3.- Representación canónica para variables en tallos según los distintos factores 


\section{INTERPRETACIÓN DE LOS EJES CANÓNICOS Y EVALUACIÓN DE LA INFLUENCIA DE LOS FACTORES.}

La valoración de la influencia de los distintos factores en base a una interpretación de los ejes canónicos requiere, vista ya la representación canónica, evaluar correlaciones entre los principales ejes significativos y las primitivas variables. El cálculo de estas correlaciones se ha llevado a cabo, matemáticamente, con la expresión

$$
r\left(w_{j}, v_{i}\right)=\frac{a_{i j}^{-1}}{\left|v_{i}\right|}
$$

siendo $\mathrm{W}_{\mathrm{j}}$ el eje canónico considerado que haya resultado significativo.

$\mathrm{V}_{\mathrm{i}}$ la variable primitiva para la que se calcula la correlación.

$\mathrm{a}_{\mathrm{ij}}^{-1}$ el elemento de fila i columna $\mathrm{j}$ de la matriz de autovectores invertida en el análisis canónico.

$\left|V_{\mathrm{i}}\right|$ la norma o desviación típica de la variable primitiva estimada a partir de la matriz básica.

El cálculo mediante (3), (tabla 16), permite obtener una interpretación de los ejes canónicos enfocada a una discriminación de los diferentes niveles, existentes en cada factor, en función de las variables establecidas.

La tabla 16 contiene doble valor de correlación para el factor época en aquellas variables cuyo análisis dio dos ejes significativos, primera columna eje representado en abcisas, segunda columna eje representado en ordenadas. Las variables que a su vez no presentan ningún valor de correlación son las que han sido excluidas en el análisis debido a la consideración (1).

DISCUSIÓN.

Influencia de la variación estacional. 
La fecha interviene de manera muy significativa sobre los contenidos en elementos minerales y pigmentos clorofílicos de las hojas de Withania frutescens y en elementos minerales de los tallos de la misma.

Las variaciones en contenidos de pigmentos de las hojas se extienden a lo largo de las seis fechas de muestreo ya indicadas. Dicha variación hace destacar un predominio de las clorofilas a y b frente a los carotenos al comienzo de la época vegetativa, y una disminución de dichas clorofilas conforme nos aproximamos a la caída de las hojas. La ordenación de los niveles es gradual a partir de Abril, esto es Abril, Junio, Julio y Agosto. Es importante destacar que las épocas de Noviembre y Febrero corresponden a una fase de formación de los órganos de la especie y por tanto presentarán una cierta inestabilidad en el contenido de los diferentes elementos y también de pigmentos. Por el contrario Abril representa la fecha en la cual se ha alcanzado el máximo desarrollo de la hoja frente al tallo, comenzando su envejecimiento paulatino hasta final de verano donde la pierde totalmente; de ahí que los extremos de variación en pigmentos correspondan a los meses de Abril y Agosto. Abril se destaca como de máximo desarrollo fisiológico de la especie.

En lo que respecta a las variaciones en contenidos de elementos minerales de las hojas, éstas contribuyen a una ordenación de los niveles como lo hacían en pigmentos: Abril, Junio, Julio, Agosto. Noviembre y Febrero aparecen intermedias en esta ordenación. Haciendo referencia a las épocas extremas podemos asociarles las siguientes características, Abril exhibirá altos contenidos en el índice V.P.F. (I.P.F.) y en elementos potasio y fósforo. En Agosto las muestras albergan altos contenidos de Magnesio (Calcio). Entre paréntesis se indican aquellas variables que no estudiadas directamente están correlacionadas positivamente con las sí estudiadas (tablas 4 y 5 ) y en consecuencia sus comportamientos serán análogos.

Existen unos elementos, $\mathrm{H}_{2} \mathrm{O}(\mathrm{N}$ y Na), cuyo máximo contenido no hace referencia a una época extrema, y que presentarán un rango de variación durante los niveles establecidos.

Finalmente el comportamiento de los tallos, ante la discriminación de los niveles, vuelve a hacer uso del mismo orden, Abril, Junio, Julio, Agosto. Abril con altos contenidos en $\mathrm{H}_{2} \mathrm{O}(\mathrm{P}, \mathrm{K}, \mathrm{y} \mathrm{Na})$ y Agosto en $\mathrm{N}, \mathrm{Ca}$ y $\mathrm{Mg}$. 
Los estudios de variaciones de pigmentos son más frecuentes a nivel de comunidades, prados, bosques, fitoplanton. Los resultados encontrados en esta especie son paralelos a las variaciones que sufre un cultivo de fitoplanton desde su siembra hasta su envejecimiento (Margalef, 1974).

Las variaciones de los contenidos de elementos en hojas son bastante acordes con los datos existentes en la bibliografía. Así Guha y Mitchell (1966) en una serie de caducifolios otoñales encuentran una disminución desde Mayo a Octubre de fósforo y potasio, un claro aumento para calcio y un ligero aumento final en magnesio y fluctuaciones en sodio. Respecto al contenido hídrico encuentra un mínimo para Julio.

Leroy (1968) para el estudio detallado de una sola especie de roble (Quercus pedunculata) pone de manifiesto una disminución del contenido en agua durante el desarrollo de la época vegetativa y así mismo disminuciones de $\%$ de $\mathrm{N}, \mathrm{P}$ y $\mathrm{K}$ y aumento de $\mathrm{Ca}$.

Por su parte Denaeyer-De Smet (1971), también para una serie de árboles caducifolios, cita un mayor contenido de N, P y K en hojas jóvenes y su disminución en las hojas adultas y al contrario, un aumento progresivo de $\mathrm{Ca}$ mientras que para el $\mathrm{Mg}$ no encuentra apenas variaciones.

Influencia de los factores altura y exposición.

La altura o posición del muestreo y su orientación al sol también intervienen de manera significativa sobre los contenidos totales de $W$. frutescens tanto en hojas como en tallos.

Para hojas el análisis ha puesto de manifiesto dos posiciones bien diferenciadas, zona alta y zona baja. La zona media presenta un solapamiento de su región con la zona baja, lo que lleva a considerar los dos niveles como uno, figuras $1 . \mathrm{b}$ y 2 .b.

Los pigmentos se reparten en esos niveles de la siguiente manera, las zonas bajas dominan en contenidos de clorofilas y las zonas altas en carotenoides. El resto de variables estudiadas en hoja se reparte de una forma más radical, la parte baja retiene mayoritariamente a los elementos $\mathrm{N}, \mathrm{P}, \mathrm{K}, \mathrm{Ca}, \mathrm{Mg}$. y contenido hídrico y la parte alta los índices 
IPF y VPF y curiosamente el elemento sodio, lo que podría caracterizar en principio la influencia de un entorno costero.

Los tallos, aun siendo más complejos en comportamiento, presentan muy bien diferenciados los tres niveles. Por una parte los tallos de las zonas bajas serán muy ricos en calcio, los de las medias en $\mathrm{P}, \mathrm{N}$ y $\mathrm{Mg}$ y los de las altas en $\mathrm{K}$, Na y contenido hídrico.

Los resultados relativos a las variaciones de concentración de elementos para exposición caracterizan perfectamente dos tipos de orientaciones: sombra y sol.

Para las hojas, los pigmentos clorofílicos y los elementos $\mathrm{N}, \mathrm{Ca}$, $\mathbf{M g}, \mathbf{K}, \mathbf{P}$ y agua, se encuentran en mayor abundancia en las muestras seleccionadas de sombra. Carotenoides, índices IPF, VPF y $\mathrm{Na}$ lo están en las de sol.

El análisis canónico ha dejado patente un paralelismo entre los factores altura y exposición.

Los tallos en general realizan una acumulación de elementos en sus muestras de sol, concretamente $\mathrm{N}, \mathrm{K}, \mathrm{Mg}, \mathrm{Na}$, Agua, quedando más influenciadas las muestras de sombra por el Ca y P.

En la distribución de clorofilas según la altura de las ramas Vanseveren y Ambroes (1971), en un estudio sobre el roble pedunculado, haya y carpe, encuentran siempre mayor cantidad de pigmentos (mg./g. materia seca) en las partes bajas de los árboles, con una relación de contenidos entre partes bajas y altas de $1.30,1.60$ y 2.17 respectivamente; nosotros para $W$. frutescens encontramos una relación aproximada de 1.59 .

Respecto al contenido de agua y elementos, según la altura y orientación, Leroy (op. cit.) concluye que las hojas de la zona baja, en parte por lo tanto de la zona menos soleada, presenta mayor contenido en agua y también en elementos minerales salvo para el potasio, que en nuestro caso no se comporta de forma excepcional en las primeras tres observaciones pero sí en las tres restantes. 


\section{BIBLIOGRAFÍA}

CUADRAS, C. M. (1974). Análisis Estadístico Multivariante y Representación Canónica de Funciones Estimables. Secretariado de Publicaciones de la Universidad de Barcelona, $23 \mathrm{pp}$. Ph. D. Summary.

DENAEYER-DE SMET, S. (1971). Teneurs en éléments biogènes des tapis végétaux dans les forêts caducifoliées d'Europe. Productivité des écosystèmes forestiers. Actes Coll. Bruxelles, Unesco.

GUHA, M. M. y MITCHELL, R. L. (1966). The trace and major element composition of the leaves of some deciduous trees. II Seasonal changes, Plant and soil. XIV 1: 90-111.

KRAMER, T y T. KOZLOWSKI (1979). Physiology of woody plants. Academic press. New York.

LEROY, P. (1968). Variations saisonnières des teneurs en eau et éléments minéraux des feuilles de chêne (Quercus Pedunculata). Ann. Sci. forest, 25 (2): 83-117.

MARGALEF, R. (1974). Ecología. Ed. Omega, Barcelona.

MARTÍN, J. y ESCARRÉ, A. (1980). Datos de fenología, reducción de hoja y variaciones estacionales de la composición foliar en cuatro especies del matorral costero del mediterráneo meridional ibérico. Mediterranea 4: 69-87.

VANSÉVEREN, J. P. y AMBROES, P. (1971). Note sur les quantités de chlorophylle à l'hectare et l'index foliaire. Bull. Soc. Bot. Belg, vol 104, 297-306. 